\title{
The Impact of US FDA and Health Canada Warnings Related to the Safety of High-dose Simvastatin
}

\author{
Kanika Anand $^{1} \cdot$ Ingrid Sketris $^{2} \cdot$ Ying Zhang $^{1} \cdot$ Adrian Levy $^{3} \cdot$ John-Michael Gamble $^{4}$
}

Published online: 27 September 2017

(c) The Author(s) 2017. This article is an open access publication

\begin{abstract}
Introduction Between 2010 and 2012, the US Food and Drug Administration and Health Canada issued warnings to healthcare professionals emphasizing the increased risk of muscle problems with high-dose simvastatin.

Objective To measure the impact of the Health Canada safety warning regarding dose-dependent adverse effects of simvastatin on prescribing of low, medium, and high doses of simvastatin.

Methods An interrupted time-series design was used to evaluate the impact of a Health Canada safety warning on 7 November 2012 regarding the safety of high-dose simvastatin. Monthly prescription records were analyzed for beneficiaries of the Nova Scotia Seniors' Pharmacare Program aged 65 years or older who had received $>1$ prescription of simvastatin between 1 January 1997 and 31 March 2015. Autoregressive Integrated Moving Average models were used to test changes in the proportion of beneficiaries dispensed a low dose $(<40 \mathrm{mg})$, medium dose (40 mg to $<80 \mathrm{mg}$ ), or high dose $(\geq 80 \mathrm{mg}$ ) of simvastatin over time.
\end{abstract}

Ingrid Sketris

ingrid.sketris@dal.ca

1 Department of Statistics, Acadia University, Wolfville, NS, Canada

2 Faculty of Health Professions, College of Pharmacy, Dalhousie University, 5968 College Street, PO Box 15000, Halifax, NS B3H 4R2, Canada

3 Department of Community Health and Epidemiology, Dalhousie University, Halifax, NS, Canada

4 School of Pharmacy, Memorial University of Newfoundland, St. John's, NL, Canada
Results There were 219 monthly periods, of which 29 periods occurred after the Health Canada warning. On average during the pre-warning periods there were 2944 simvastatin users per month, of whom $71 \%$ were dispensed a low dose, $26 \%$ a medium dose, and $2 \%$ a high dose. The proportion of beneficiaries dispensed low-dose simvastatin increased by $0.9 \%$ (one-sided $p$ value $0.035 ; 90 \%$ CI $0.07-1.65$ ), the proportion dispensed medium-dose simvastatin decreased by $0.7 \%$ (one-sided $p$ value $0.0496 ; 90 \%$ CI -1.48 to -0 ), and there was no significant change in the proportion dispensed high-dose simvastatin $(-0.15 \%$ change, one-sided $p$ value 0.205 ; $90 \%$ CI -0.45 to 0.15 ). Conclusions The Health Canada Health Care Professional warning had a small effect on increasing the proportion of beneficiaries dispensed low and medium doses of simvastatin but not high doses of simvastatin. Nevertheless, there remain seniors in Nova Scotia receiving high-dose simvastatin for whom the benefit/risk potential may need to be re-evaluated.

\section{Key Points}

The Health Canada warning related to increased risk of muscle problems associated with high-dose simvastatin had a small effect on increasing the proportion of beneficiaries dispensed low and medium doses of simvastatin but not on decreasing the proportion receiving high doses of simvastatin.

Due to the limited effect of "Dear Health Care Professional" letters, other intervention strategies are needed to increase the awareness and the uptake of recommendations related to simvastatin dosage to improve the risk/benefit of prescribing statins. 


\section{Introduction}

Statin medications are useful for prevention and treatment of cardiovascular disease and stroke [1-4]. The evidence on the effectiveness of statins from randomized controlled trials and meta-analyses [4-6] supports the strong recommendations in clinical practice guidelines, which has ultimately led to their widespread use [7]. Prescriptions for statins are increasing in Canada, especially in older adults [8-17].

Although statins are relatively well tolerated, studies have documented the types and prevalence of adverse effects of statins, particularly in high doses. Adverse muscle-related effects are relatively common, occurring in $10-30 \%$ of patients [18-20] and may be dose related; however, severe myopathies with clinically relevant elevation in muscle enzymes occur in less than $1 \%$ of patients [21]. In addition, other adverse effects have been reported, such as an increased risk of diabetes and acute kidney injury [22-24].

As the published literature and other evidence on the adverse effects of medications are accumulated post-marketing, regulatory authorities have developed mechanisms to communicate these potential adverse effects with prescribers. A common approach is the use of "Dear Health Care Professional" (i.e., "Dear Doctor") letters, which identify the nature and magnitude of the risk and may be sent by mail, fax, or electronically. The use of these safety communications has been criticized as their impact on changing prescribing has been limited. A systematic review by Piening et al. [25] examined the impact of safety-related regulatory action on clinical practice and concluded that many of the 50 identified articles lacked rigor in study design and statistical analysis. Of these studies, few were from Canada [26-29]. One study evaluated the effect of an alerting system on the prescribing of statins and macrolide antibiotic co-prescriptions, but none were specifically related to simvastatin [30].

Numerous healthcare professional alerts have been issued globally regarding the safety of high-dose simvastatin and an increased risk of myopathy, including rhabdomyolysis. Specifically, the US Food and Drug Administration (FDA) issued a warning to increase the awareness of rhabdomyolysis with high-dose simvastatin on 19 March 2010 [31]. The FDA issued an additional warning on 8 June 2011 that suggested no new patients should be started on $80 \mathrm{mg}$ and should maintain an $80-\mathrm{mg}$ dose only if they had been on this for 12 months in the absence of muscle toxicity [32]. The UK Medicines and Healthcare Products Regulatory Agency (MHRA) issued a warning on 1 May 2010 related to high-dose simvastatin [33]. The European Medicines Agency (EMA) has also issued recommendations regarding dose-related adverse events of simvastatin [34]. Health Canada followed on 7 November 2012 [35], and suggested a recommended daily dose of simvastatin of $\leq 40 \mathrm{mg}$. A major impetus for these alerts stems from the findings of the Study of the Effectiveness of Additional Reductions in Cholesterol and Homocysteine (SEARCH) trial [36]. The incidence of confirmed myopathy was substantially higher in patients randomized to simvastatin $80 \mathrm{mg}(1 \%)$ compared to $20 \mathrm{mg}$ $(\sim 0.02 \%)$ daily [relative risk $26.6,95 \%$ confidence interval (CI) 6.5-109.3] [36].

The impact of the Health Canada warning on statin prescriptions is unknown. Other studies have shown limited impact if direct healthcare professional communications are used alone without other interventions, but the impact varies by drug, level of risk, and other factors [37]. The Nova Scotia Department of Health and Wellness did not have a specific intervention related to statin prescribing in conjunction with the Health Canada warning. This study estimates changes from January 1997 to March 2015 in the proportion of Nova Scotia Seniors' Pharmacare Program (NSSPP) beneficiaries receiving prescriptions for low-, medium-, and high-dose simvastatin.

\section{Methods}

\subsection{Study Population}

The study cohort was derived from NSSPP beneficiaries. Nova Scotia is a Canadian province with a population of about 940,000 residents [38]. Physician services are provided, without charge, for insured services from approximately 1100 family physicians and 1100 medical specialists [39]. The source population comprised of all patients aged 65 years or older registered for Nova Scotia medical services coverage who enrolled and paid the required premiums and co-payments for the NSSPP and received at least one prescription of simvastatin between 1 January 1997 and 31 March 2015. The NSSPP does not provide drug insurance to residents insured by private drug programs or other public sector drug programs such as Veterans Affairs Canada or First Nations and Inuit Health. The NSSPP reimbursed all statins marketed in Canada, including atorvastatin, rosuvastatin, simvastatin, fluvastatin, lovastatin, and pravastatin. Cerivastatin was removed from the market and is not included in the study.

Ethics approval was received from the Dalhousie University Ethics Committee (effective date 8 July 2014; renewal 8 July 2015).

\subsection{Study Design}

We used an interrupted time-series design, which is the strongest quasi-experimental study design to examine changes following an intervention on a target population 
using observational data [40]. Specifically, this design includes the pre-intervention time period as a control and post-intervention periods as treatments. Assuming a trendstationary model (deterministic trend with stationary error process) with a reasonable number of observations in the pre-intervention period, an adequate model can be identified without requiring a large number of observations in the post-intervention period [41]. This approach has been previously used with Nova Scotia Pharmacare prescription data $[42,43]$.

We examined the monthly proportion of beneficiaries of dispensed prescriptions for simvastatin high-dose ( $\geq 80 \mathrm{mg} /$ day), medium-dose $(40 \mathrm{mg} /$ day to $<80 \mathrm{mg} /-$ day), and low-dose $(<40 \mathrm{mg} /$ day) before and after multiple healthcare professional regulatory warnings. The rationale for categorizing all doses less then $40 \mathrm{mg}$ as low dose and medium dose as $40 \mathrm{mg}$ was to capture changes in dosage from 80 to $40 \mathrm{mg}$ of simvastatin given the Health Canada warning recommended a simvastatin dosage of 5-40 mg. The proportion of beneficiaries was obtained by dividing the monthly beneficiary count by the total NSSPP beneficiaries for a given month. Furthermore, the proportion of beneficiaries per month data was first divided into four time periods: (1) pre-warning or pre-intervention period (January 1997 to February 2010); (2) FDA I period (March 2010 to May 2011); (3) FDA II period (June 2011 to October 2012); and (4) Health Canada period (November 2012-March 2015).

Given Health Canada's warning regarding the potential risks of high-dose simvastatin, our primary aim was to test changes in the proportion of beneficiaries per month receiving simvastatin doses greater than or equal to $80 \mathrm{mg}$ simvastatin before and after the Health Canada regulatory warning on 7 November 2012. However, it is plausible that prescribers in Nova Scotia may be responsive to warnings issued by the FDA and therefore tested for the incremental change in the proportion of high-, medium-, and low-dose simvastatin users after the FDA I and FDA II periods. To our knowledge, there were no other major Nova Scotia provincial-level interventions related specifically to simvastatin dosing during the investigated time period, other than those considered.

\subsection{Statistical Analysis}

To test changes in the proportion of beneficiaries dispensed low-, medium-, and high-dose simvastatin we employed a time series regression model by applying a two-stage modeling approach. This approach accounts for long-term time effects and other time-related covariate/factor effects as well as serially random fluctuations. Both long-term trend and the seasonal effects can be modelled by either deterministic or random effects as necessary. By controlling any effects from FDA warnings, any change detected between the FDA I and II and Health Canada periods would thus likely be associated with the Health Canada regulatory warning (intervention) effect.

In the first stage, a linear regression model was fitted with co-variates, stepwise variables corresponding to invention time periods to examine the FDA warnings and the impact of the Health Canada warning letter. The residuals from this initial model were examined to check the possible autocorrelation for dependence. If the significant autocorrelation was found in the residuals then a suitable Auto-Regressive Integrated Moving Average (ARIMA) model or seasonal ARIMA model was determined for the residual process using the Akaike Information Criterion (AIC). The final model was a regression with an ARIMA error model identified by the residual analysis, which includes checking of autocorrelation function (ACF), partial autocorrelation function (PACF), and normality of residuals [44]. This procedure was followed on one dosage series at a time.

The statistical program used was R 3.1.1 [45].

\section{Results}

There were a total of 219 time intervals, of which 158 intervals were in the pre-warning period ( $\sim 73 \%$ of data values), 32 time intervals in the FDA I and II periods (15 time intervals in the FDA I period ( $\sim 7 \%$ of data values), 17 time intervals in the FDA II period ( $\sim 8 \%$ of data values)), and 29 time intervals after the Health Canada warning ( $\sim 13 \%$ of data values). On average in the prewarning period, there were a total of 2944 simvastatin users per month, of whom approximately $71 \%$ were dispensed a low dose, $26 \%$ were dispensed a medium dose, and $2 \%$ were dispensed a high dose. Table 1 provides descriptive statistics of monthly proportions of three dose categories for the pre-warning, FDA I, FDA II, and Health Canada periods. Briefly, on average across all time periods, over $50 \%$ of simvastatin users were dispensed a low dose, between 23 and 39\% a medium dose, and less than 5\% a high dose.

Figure 1 shows the total NSSPP simvastatin beneficiary count per month over a period of 18 years between 1 January 1997 and 31 March 2015. The interventions are denoted by the vertical colored lines. Clearly Fig. 1 shows the total patient count receiving simvastatin per month was changing over time. With this total count for a given month as the denominator, Fig. 2 shows the three times series proportions of the low, medium, and high dose for a given month with the intervention depicted by vertical lines. These plots show a long-term trend in all three series with some subtle increase in the low-dose series and a slight 
Table 1 Descriptive statistics for the percentage of Nova Scotia Seniors' Pharmacare Program (NSSPP) beneficiaries dispensed (a) low dosage $(<40 \mathrm{mg})$, (b) medium dosage ( $\geq 40 \mathrm{mg}$ or $<80 \mathrm{mg}$ ), and (c) high dosage ( $\geq 80 \mathrm{mg}$ ) simvastatin

\begin{tabular}{|c|c|c|c|c|c|c|c|c|}
\hline Time period & $\begin{array}{l}\text { Number of months } \\
(N)\end{array}$ & $\begin{array}{l}\text { Minimum } \\
(\%)\end{array}$ & $\begin{array}{l}25 \text { th } \\
\text { percentile }\end{array}$ & $\begin{array}{l}\text { Median } \\
(\%)\end{array}$ & $\begin{array}{l}\text { Mean } \\
(\%)\end{array}$ & $\begin{array}{l}\text { Standard } \\
\text { deviation }\end{array}$ & $\begin{array}{l}75 \text { th } \\
\text { percentile }\end{array}$ & $\begin{array}{l}\text { Maximum } \\
(\%)\end{array}$ \\
\hline $\begin{array}{l}\text { (a) Pre- } \\
\text { warnings }\end{array}$ & 158 & 56.5 & 64.4 & 71.6 & 75.3 & 13.1 & 88.4 & 97.0 \\
\hline FDA I & 15 & 55.8 & 56.4 & 56.6 & 56.6 & 0.4 & 56.8 & 57.0 \\
\hline FDA II & 17 & 54.8 & 55.4 & 55.7 & 55.8 & 0.5 & 56.2 & 57.0 \\
\hline $\mathrm{HC}$ & 29 & 55.1 & 57.3 & 57.6 & 57.5 & 0.7 & 57.9 & 59.0 \\
\hline $\begin{array}{l}\text { (b) Pre- } \\
\text { warnings }\end{array}$ & 158 & 2.9 & 10.8 & 26.0 & 22.8 & 11.9 & 33.2 & 39.0 \\
\hline FDA I & 15 & 38.1 & 38.7 & 38.8 & 38.8 & 0.4 & 38.9 & 39.0 \\
\hline FDA II & 17 & 38.8 & 39.3 & 39.6 & 39.6 & 0.5 & 39.8 & 41.0 \\
\hline $\mathrm{HC}$ & 29 & 37.7 & 38.3 & 38.7 & 38.8 & 0.6 & 39.1 & 41.0 \\
\hline $\begin{array}{l}\text { (c) Pre- } \\
\text { warnings }\end{array}$ & 158 & 0.0 & 0.9 & 2.3 & 1.9 & 1.2 & 2.7 & 4.0 \\
\hline FDA I & 15 & 4.1 & 4.5 & 4.7 & 4.7 & 0.2 & 4.8 & 5.0 \\
\hline FDA II & 17 & 4.0 & 4.3 & 4.7 & 4.6 & 0.3 & 4.8 & 5.0 \\
\hline $\mathrm{HC}$ & 29 & 3.1 & 3.5 & 3.7 & 3.8 & 0.4 & 4.1 & 5.0 \\
\hline
\end{tabular}

FDA US Food and Drug Administration, HC Health Canada, NSSPP Nova Scotia Seniors' Pharmacare Program, SD standard deviation

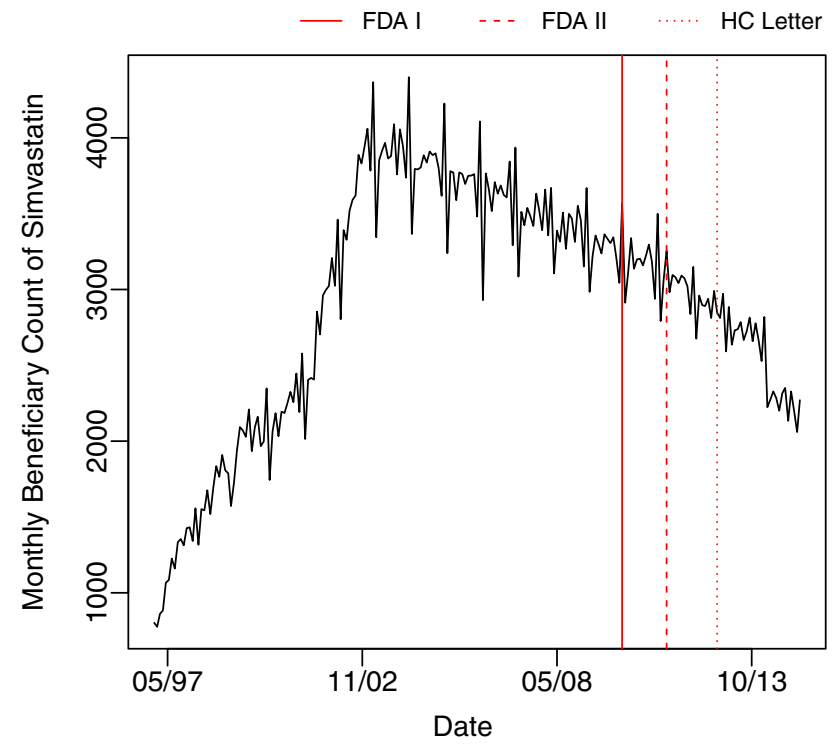

Fig. 1 Monthly beneficiary counts of simvastatin prescription records for eligible Nova Scotia Seniors' Pharmacare Program (NSSPP) beneficiaries, January 1997 to March 2015. FDA US Food and Drug Administration, NSSPP Nova Scotia Seniors' Pharmacare Program

decrease in the medium- and high-dose series after the Health Canada warning, which can be similarly observed in Table 1, the descriptive statistics.

The long-term trend and the serially autocorrelated random fluctuations in the time series of the low-dose beneficiary proportion data were modelled by an ARIMA $(5,1,0)$. A regression model with intervention factors of the FDA's and Health Canada's warnings gave reasonable

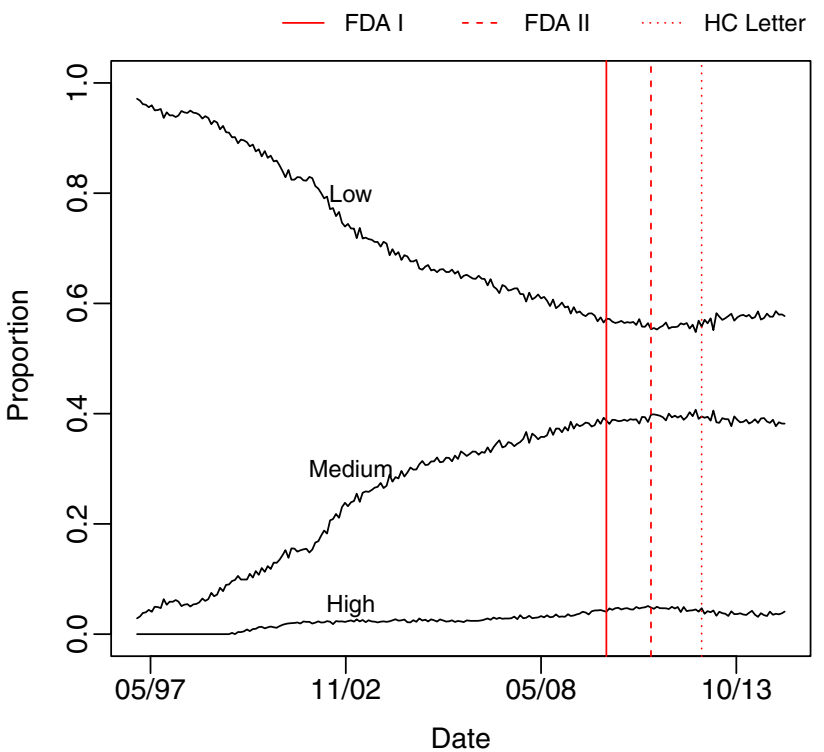

Fig. 2 Monthly proportion of Nova Scotia Seniors' Pharmacare Program (NSSPP) beneficiaries dispensed low $(<40 \mathrm{mg} /$ day $)$, medium ( $\geq 40 \mathrm{mg} /$ day and $<80 \mathrm{mg} /$ day $)$, and high ( $\geq 80 \mathrm{mg} /$ day $)$ of simvastatin, January 1997 to March 2015. The red vertical lines indicate timing of safety alert (FDA I: 19 March 2010; FDA II: 8 June 2011; Health Canada: 7 November 2012). FDA US Food and Drug Administration, NSSPP Nova Scotia Seniors' Pharmacare Program

intervention estimates, while incorporating the dependent structure of the ARIMA $(5,1,0)$ model. The residual diagnostic analysis confirmed the final model adequacy. Similarly, a regression with a dependent-model ARIMA $(13,1,0)$ was applied for the medium-dosage time series. The residual analysis presented no significant anomalies 
confirming the model adequacy. For the high-dosage series an ARIMA $(4,1,0)$ model was selected to model the longterm and serially time-related autocorrelation in the data since November 1999 because no patient received a high dose before that time. The residual analysis presented some anomalies, which may indicate the evidence of lack of fit. One reason for the model inadequacy might be the complex variations arising during the intervention time period. The model should be interpreted with caution.

The results from the time series regression model described above show that the proportion of beneficiaries receiving lowdose simvastatin increased by $0.9 \%$ after the Health Canada warning (one-sided $p$ value 0.035 ; 90\% CI 0.07-1.65) suggesting an overall increase in proportion of beneficiaries dispensed a low dose. On the contrary, the proportion of beneficiaries receiving a medium dose decreased by $0.7 \%$ after the Health Canada warning (one-sided $p$ value 0.0496; $90 \% \mathrm{CI}-1.48$ to -0 ). No significant change was observed in the proportion of high-dose users after the Health Canada warning ( $-0.15 \%$ change, one-sided p value $0.205 ; 90 \% \mathrm{CI}-$ 0.45 to 0.15$)$. By March 2015, less than 5\% $(n=93)$ of beneficiaries were receiving $80 \mathrm{mg}$ of simvastatin.

\section{Discussion}

The "Dear Health Care Professional" letter issued by Health Canada on 7 November 2012 related to high-dose simvastatin was not associated with any significant change in the proportion of NSSPP beneficiaries receiving $\geq 80 \mathrm{mg}$ daily dose of simvastatin. However, there was a small statistically significant increase $(<1 \%)$ in the proportion of low-dose statin users and decrease in the proportion of medium-dose statin users. Importantly, our findings raise concerns about the effectiveness of the regulatory warnings to quickly change prescribing.

Our study tested the incremental change in the proportion of simvastatin high-, medium-, and low-dose users of the Health Canada warning following other regulatory warnings. Although the Health Canada warning in November of 2012 emphasized the potential harms of highdose simvastatin, the potential harms of $80 \mathrm{mg}$ doses had already been communicated by US and European drug regulatory agencies. Therefore, the additional impact of another regulator may be negligible. Furthermore, physicians may be unaware of the regulatory warnings, disagree with the warnings, or disregard the warnings as they may feel their patients have been stable for some time.

Another potential explanation for our findings are that the number of patients dispensed high-dose statins represents a small proportion of users, thereby minimizing the power of our analysis to detect a change in this group. By March 2015 only 93 NSSPP beneficiaries (4\% of NSSPP statin users) were receiving a high simvastatin dose, whereas approximately $38 \%$ of NSSPP statin users were receiving a medium dose and approximately $58 \%$ of beneficiaries were dispensed a low dose of simvastatin following the Health Canada warning.

Our findings are consistent with previous studies and those included in systematic reviews that note that government drug regulatory agencies' passive risk communications advisories have a modest effect on changing drug prescribing and monitoring [25]. Studies examining the effect of FDA and EMA warnings found variable effect sizes of regulatory warnings $(2-50 \%)$ [25, 46-52]. However, the studies note that the effects are variable depending on such factors as the type of physicians targeted, the type of drug, the nature and frequency of risk, patient characteristics, the amount of scientific certainty, supporting documentation from specialist physicians, and the amount of media attention received. Some drug risk communications are associated with a large and fast impact on drug prescribing and monitoring, while others have no impact, more modest impacts, or delayed impacts [46, 48, 49, 52-56]. Many studies had inadequate designs $[25,57,58]$ and some studies note that passive dissemination strategies to improve prescribing behaviors have been shown to be modestly successful, with effect sizes in the range of $8 \%[59,60]$.

\subsection{Strengths and Limitations}

This study had a number of strengths. It used longitudinal data, representing over $80 \%$ of Nova Scotia's older adults, since the majority are enrolled in Pharmacare (Health Data Nova Scotia, personal communication). It involved both urban and rural and academic and non-academic practice settings. We used the Box and Tiao method [44], which has been found to be useful for a limited number of time points.

There are also some limitations to our study. The data set used has only NSSPP beneficiaries and excludes those patients covered by private insurance or other publicly funded drug programs, those paying out-of-pocket, or those receiving prescription drug samples. Prescriptions for residents in nursing homes are included in the data and not reported separately. We are unable to capture prescriptions while patients are in hospital. We examined all prescriptions and did not identify newly started prescriptions separately. Although regulatory health warnings, including the Health Canada warning in November 2012, recommended that no patients initiate simvastatin $80 \mathrm{mg}$, the warning also pertained to those with ongoing simvastatin $80 \mathrm{mg}$ doses; therefore, we decided to include all NSSPP beneficiaries receiving a statin prescription. Furthermore, given the small number of new users, we did not stratify our sample into incident and prevalent users. We examined the 
effect of the Health Canada warnings but physicians may also have been influenced by many sources, including international literature, pharmaceutical industry marketing approaches, media, direct-to-consumer advertising, patient coupons for statins, etc. [52]. We were unable to determine if the physician was unaware of the warning, chose to exceed the dosage in the warning due to a specific benefit/ risk assessment, or was aware of the warning but determined that the evidence in the warning was limited [61]. We were unable to determine if prescribers decreased simvastatin dosages for some individuals from a high to a medium dose. Moreover, we did not determine if the small changes in the proportion of statin users using low and medium doses were due to patients switching doses of simvastatin or due to patients switching to alternative statins.

Even though our study examined 29 months of data after the Health Canada warning, physicians had access to published scientific literature related to the association between high-dose simvastatin and myopathy several years before the FDA and Health Canada warnings [62]. We did not determine the risks and benefits of statin therapy for individual patients including co-morbidities, co-prescribing, and the duration of statin therapy, or patient health outcomes. Although beyond the scope of this study, it is important to further study patients who may be receiving sub-optimal simvastatin doses such as those at high risk of a cardiovascular event. We were unable to determine the healthcare system, provider, and patient factors that contributed to the relatively slow uptake of the regulatory warnings. Physicians may have been treating patients to specific target low density lipoprotein concentrations and increasing the dose of simvastatin to reach that target [46].

Further work is needed to assist physicians in determining the potential harms, benefits, and uncertainties of prescribing for individual patients, including approaches to presenting the quality of evidence and the magnitude of the risks [61, 63, 64]. Multicomponent interventions including computerized decision support and personalized audit and feedback may have a greater effect than passively disseminated "Dear Healthcare Professional" letters [65]. However, one study examining the impact of computerized alerts related to medication black-box warnings in ambulatory care had limited effect [66]. Approaches have been taken to increase the effectiveness of the regulatory warnings. A study by Reber et al. [37] examined the characteristics determining the impact of the Direct Health Care Professional Communications in The Netherlands and found that a standard template emphasizing the drug safety issues at hand and the type of serious safety issues were associated with changes in new drug use [37].

\section{Conclusion}

Statin medications in high doses have the potential to increase the risk of adverse effects. The Health Canada warning in 2012 was associated with a small, statistically significant change in the proportion of beneficiaries dispensed low and medium doses but not high doses of simvastatin. Prior warnings from other regulatory agencies may have diminished the impact of the Health Canada warning. Importantly, there remain older adults in Nova Scotia receiving $80 \mathrm{mg}$ or more of simvastatin for whom the benefit/risk potential may need re-evaluation. Further research on methods to increase the awareness and prescribing changes recommended by regulatory warnings is needed, including incorporation into clinical decision support systems.

Acknowledgements We would like to thank Health Data Nova Scotia of Dalhousie University for the data used in this study; the Canadian Network for Observational Drug Effect Studies (CNODES), a collaborating center of the Drug Safety and Effectiveness Network (DSEN) and funded by the Canadian Institutes of Health Research (Grant number DSE-111845), for data access and support; Hala Tamim for assistance with data access procedures; and Kim Kelly and Pam McLean Vesey for literature review. Kanika Anand is supported by the Nova Scotia Health Research Foundation (NSHRF) student support grant and JM Gamble is supported by a CIHR New Investigator Award and a Clinician Scientist Award from the Canadian Diabetes Association. We would like to thank Hala Tamim and Robyn Traynor for review of the manuscript, and Bill Kidney, Anja Haltner, and Sota Ichiba for preparation of the preliminary analysis and the $\mathrm{R}$ computing code.

Role of investigators Conception and design of the project: All investigators. Acquisition of data: Dr. AL. Statistical analysis of the data: Dr. YZ. Analysis and interpretation of data: All investigators with input related to specific areas of expertise: Dr. AL: pharmacoepidemiology, data quality; Dr. YZ: statistics; Dr. IS: drug use management and policy, knowledge translation; Dr. J-MG: epidemiology, statistical analysis; and pharmacotherapy; and KA: data analysis. All investigators contributed to the drafting of the manuscript, including critical revision of the manuscript for important intellectual content.

Disclaimer and Compliance with Ethical Standards The data (or portions of the data) used in this report were made available by Health Data Nova Scotia of Dalhousie University. Although this research is based on data obtained from the Nova Scotia Department of Health and Wellness, the observations and opinions expressed are those of the authors and do not represent those of either Health Data Nova Scotia or the Department of Health and Wellness. This study was approved by the Dalhousie University Health Sciences Research Ethics Board (REB \# 2014-3269).

Funding Ingrid Sketris has received salary support, in part, from the Canadian Institutes of Health Research for the CNODES project.

Conflicts of interest The authors (KA, IS, YZ, AL, JMG) have no conflicts of interest to declare.

Open Access This article is distributed under the terms of the Creative Commons Attribution-NonCommercial 4.0 International 
License (http://creativecommons.org/licenses/by-nc/4.0/), which permits any noncommercial use, distribution, and reproduction in any medium, provided you give appropriate credit to the original author(s) and the source, provide a link to the Creative Commons license, and indicate if changes were made.

\section{References}

1. Wilson PW. Established risk factors and coronary artery disease: the Framingham study. Am J Hypertens. 1994;7(7 Pt 2):7S-12S.

2. Tanuseputro P, Manuel DG, Leung M, Nguyen K, Johansen H, Canadian Cardiovascular Outcomes Research Team. Risk factors for cardiovascular disease in Canada. Can $\mathbf{J}$ Cardiol. 2003;19(11):1249-59.

3. Antman EM, Anbe DT, Armstrong PW, Bates ER, Green LA, Hand M, Hochman JS, Krumholz HM, Kushner FG, Lamas GA, Mullany CJ, Ornato JP, Pearle DL, Sloan MA, Smith SC Jr, Alpert JS, Anderson JL, Faxon DP, Fuster V, Gibbons RJ, Gregoratos G, Halperin JL, Hiratzka LF, Hunt SA, Jacobs AK, Ornato JP. ACC/AHA guidelines for the management of patients with ST-elevation myocardial infarction: a report of the American College of Cardiology/American Heart Association Task Force on Practice Guidelines (Committee to Revise the 1999 Guidelines for the Management of patients with acute myocardial infarction). J Am Coll Cardiol. 2004;44(3):E1-211.

4. Anderson TJ, Mancini GB, Genest J Jr, Gregoire J, Lonn EM, Hegele RA. The new dyslipidemia guidelines: what is the debate? Can J Cardiol. 2015;31(5):605-12.

5. Brugts JJ, Yetgin T, Hoeks SE, Gotto AM, Shepherd J, Westendorp RG, de Craen AJ, Knopp RH, Nakamura H, Ridker P, van Domburg R, Deckers JW. The benefits of statins in people without established cardiovascular disease but with cardiovascular risk factors: meta-analysis of randomised controlled trials. BMJ. 2009;338:b2376.

6. Tonelli M, Lloyd A, Clement F, Conly J, Husereau D, Hemmelgarn B, Klarenbach S, McAlister FA, Wiebe N, Manns B, Alberta Kidney Disease Network. Efficacy of statins for primary prevention in people at low cardiovascular risk: a meta-analysis. CMAJ. 2011;183(16):E1189-202.

7. Ioannidis JP. More than a billion people taking statins?: potential implications of the new cardiovascular guidelines. JAMA. 2014;311(5):463-4.

8. Sketris IS, Kephart GC, Hicks VA, Hubbard EJ, Brown MG, Chandler RF, MacLean DR. Prescribing patterns of antilipemic drugs and prevalence of hypercholesterolemia in the Nova Scotia population more than 65 years old. Ann Pharmacother. 1995;29(6):576-81.

9. McAlister FA, Taylor L, Teo KK, Tsuyuki RT, Ackman ML, Yim R, Montague TJ. The treatment and prevention of coronary heart disease in Canada: do older patients receive efficacious therapies? The Clinical Quality Improvement Network (CQIN) Investigators. J Am Geriatr Soc. 1999;47(7):811-8.

10. Jackevicius CA, Tu K, Filate WA, Brien SE, Tu JV, Canadian Cardiovascular Outcomes Research Team. Trends in cardiovascular drug utilization and drug expenditures in Canada between 1996 and 2001. Can J Cardiol. 2003;19(12):1359-66.

11. Simpson E, Beck C, Richard H, Eisenberg MJ, Pilote L. Drug prescriptions after acute myocardial infarction: dosage, compliance, and persistence. Am Heart J. 2003;145(3):438-44.

12. Pilote L, Beck CA, Karp I, Alter D, Austin P, Cox J, Humphries K, Jackevicius C, Richard H, Tu JV, Canadian Cardiovascular Outcomes Research Team. Secondary prevention after acute myocardial infarction in four Canadian provinces, 1997-2000. Can J Cardiol. 2004;20(1):61-7.
13. Neutel CI, Morrison H, Campbell NR, de Groh M. Statin use in Canadians: trends, determinants and persistence. Can J Public Health. 2007;98(5):412-6.

14. Raymond CB, Morgan SG, Katz A, Kozyrskyj AL. A populationbased analysis of statin utilization in British Columbia. Clin Ther. 2007;29(9):2107-19.

15. Kozyrskyj A, Raymond C, Dahl M, Ekuma O, Schultz J, Sklepowich M, Bond R. The effects of Manitoba pharmacare formulary policy on utilization of prescription medications. Winnipeg: Manitoba Centre for Health Policy; 2009.

16. Canadian Institute for Health Information. Prescribed Drug Spending in Canada, 2012: A Focus on Public Drug Programs. Ottawa: Ontario CIHI; 2014. https://secure.cihi.ca/free_products/ Prescribed_Drug_Spending_in_Canada_EN.pdf. Accessed 18 Sept 2017.

17. Minard LV, Corkum A, Sketris I, Fisher J, Zhang Y, Saleh A. Trends in statin use in seniors 1999-2013: time series analysis. PLoS One. 2016;11(7):e0158608.

18. Dalhousie CME Academic Detailing Service. Lipid lowering in primary prevention: a calculated risk. 2013. http://cme.medicine. dal.ca/ad_resources.htm. Accessed 27 Feb 2017.

19. Ganga HV, Slim HB, Thompson PD. A systematic review of statin-induced muscle problems in clinical trials. Am Heart J. 2014;168(1):6-15.

20. Mancini GB, Tashakkor AY, Baker S, Bergeron J, Fitchett D, Frohlich J, Genest J, Gupta M, Hegele RA, Ng DS, Pearson GJ, Pope J. Diagnosis, prevention, and management of statin adverse effects and intolerance: Canadian Working Group Consensus update. Can J Cardiol. 2013;29(12):1553-68.

21. Kashani A, Phillips CO, Foody JM, Wang Y, Mangalmurti S, Ko DT, Krumholz HM. Risks associated with statin therapy: a systematic overview of randomized clinical trials. Circulation. 2006;114(25):2788-97.

22. Sattar N, Preiss D, Murray HM, Welsh P, Buckley BM, de Craen AJ, Seshasai SR, McMurray JJ, Freeman DJ, Jukema JW, Macfarlane PW, Packard CJ, Stott DJ, Westendorp RG, Shepherd J, Davis BR, Pressel SL, Marchioli R, Marfisi RM, Maggioni AP, Tavazzi L, Tognoni G, Kjekshus J, Pedersen TR, Cook TJ, Gotto AM, Clearfield MB, Downs JR, Nakamura H, Ohashi Y, Mizuno K, Ray KK, Ford I. Statins and risk of incident diabetes: a collaborative meta-analysis of randomised statin trials. Lancet. 2010;375(9716):735-42.

23. Dormuth CR, Filion KB, Paterson JM, James MT, Teare GF, Raymond CB, Rahme E, Tamim H, Lipscombe L, Canadian Network for Observational Drug Effect Studies Investigators. Higher potency statins and the risk of new diabetes: multicentre, observational study of administrative databases. BMJ. 2014;348:g3244.

24. Dormuth CR, Hemmelgarn BR, Paterson JM, James MT, Teare GF, Raymond CB, Lafrance JP, Levy A, Garg AX, Ernst P, Canadian Network for Observational Drug Effect Studies. Use of high potency statins and rates of admission for acute kidney injury: multicenter, retrospective observational analysis of administrative databases. BMJ. 2013;346:f880.

25. Piening S, Haaijer-Ruskamp FM, de Vries JT, van der Elst ME, de Graeff PA, Straus SM, Mol PG. Impact of safety-related regulatory action on clinical practice: a systematic review. Drug Saf. 2012;35(5):373-85.

26. Azoulay L, Oraichi D, Berard A. Patterns and utilization of isotretinoin for acne from 1984 to 2003: is there need for concern? Eur J Clin Pharmacol. 2006;62(8):667-74.

27. Katz LY, Kozyrskyj AL, Prior HJ, Enns MW, Cox BJ, Sareen J. Effect of regulatory warnings on antidepressant prescription rates, use of health services and outcomes among children, adolescents and young adults. CMAJ. 2008;178(8):1005-11. 
28. Kurdyak PA, Juurlink DN, Mamdani MM. The effect of antidepressant warnings on prescribing trends in Ontario, Canada. Am J Public Health. 2007;97(4):750-4.

29. Valiyeva E, Herrmann N, Rochon PA, Gill SS, Anderson GM. Effect of regulatory warnings on antipsychotic prescription rates among elderly patients with dementia: a population-based timeseries analysis. CMAJ. 2008;179(5):438-46.

30. Morera T, Gervasini G, Carrillo JA, Benitez J. Evaluation of a drug-drug interaction alert structure through the retrospective analysis of statins-macrolides co-prescriptions. Basic Clin Pharmacol Toxicol. 2005;96(4):289-94.

31. US Food and Drug Administration. FDA warns about increased risk of muscle injury with zocor. 2010. http://www.fda.gov/ NewsEvents/Newsroom/PressAnnouncements/ucm205215.htm. Accessed 27 Feb 2017.

32. US Food and Drug Administration. FDA announces new safety recommendations for high-dose simvastatin. 2011. http://www. fda.gov/NewsEvents/Newsroom/PressAnnouncements/ ucm258338.htm. Accessed 27 Feb 2017.

33. Medicines and Healthcare Products Regulatory Agency. Simvastatin: increased risk of myopathy at high dose $(80 \mathrm{mg})$. Drug Saf Update. 2010;3(10):7.

34. European Medicines Agency. Pharmacovigilance Risk Assessment Committee (PRAC) recommendation: HMG-CoA Reductase Inhibitors - Simvastatin - Risk of myopathy and rhabdomyolysis associated with high doses. London: United Kingdom European Medicines Agency; 2014. https://db.cbgmeb. nl/veegactie/csp/Simvastatin_april2014.pdf. Accessed 18 Sept 2017.

35. Government of Canada. Health Canada endorsed important safety information on zocor (simvastatin). 2012; http://www. healthycanadians.gc.ca/recall-alert-rappel-avis/hc-sc/2012/ 15826a-eng.php?_ga=1.181258983.15809296.1441990120. Accessed 24 Feb 2017.

36. Study of the Effectiveness of Additional Reductions in Cholesterol and Homocysteine (SEARCH) Collaborative Group, Armitage J, Bowman L, Wallendszus K, Bulbulia R, Rahimi K, Haynes R, Parish S, Peto R, Collins R. Intensive lowering of LDL cholesterol with $80 \mathrm{mg}$ versus $20 \mathrm{mg}$ simvastatin daily in 12,064 survivors of myocardial infarction: a double-blind randomised trial. Lancet. 2010;376(9753):1658-69.

37. Reber KC, Piening S, Wieringa JE, Straus SM, Raine JM, de Graeff PA, Haaijer-Ruskamp FM, Mol PG. When direct healthcare professional communications have an impact on inappropriate and unsafe use of medicines. Clin Pharmacol Ther. 2013;93(4):360-5.

38. Statistics Canada. Table 051-0005: Estimates of population, Canada, provinces and territories, quarterly (persons), CANSIM (database). 2015. http://www5.statcan.gc.ca/cansim/a26?lang= eng\&retrLang=eng\&id=0510005\&paSer $=\&$ pattern $=\&$ stByVal $=$ $1 \& \mathrm{p} 1=1 \& \mathrm{p} 2=-1 \&$ tabMode=dataTable $\&$ csid $=$. Accessed $24 \mathrm{Feb}$ 2017.

39. Canadian Institute for Health Information. Supply, distribution and migration of Canadian physicians, 2009. Ottawa: Ontario CIHI; 2010. https://secure.cihi.ca/free_products/SMDB_2009_ EN.pdf. Accessed 18 Sept 2017.

40. Wagner AK, Soumerai SB, Zhang F, Ross-Degnan D. Segmented regression analysis of interrupted time series studies in medication use research. J Clin Pharm Ther. 2002;27(4):299-309.

41. McLeod AI, Vingilis ER. Power computations for intervention analysis. Technometrics. 2005;47(2):174-81.

42. Lowe DO, Lummis H, Zhang Y, Sketris IS. Effect of educational and policy interventions on institutional utilization of wet nebulization respiratory drugs and portable inhalers. Can J Clin Pharmacol. 2008;15(2):e334-43.
43. Fisher JE, Zhang Y, Sketris I, Johnston G, Burge F. The effect of an educational intervention on meperidine use in Nova Scotia, Canada: a time series analysis. Pharmacoepidemiol Drug Saf. 2012;21(2):177-83.

44. Box GEP, Tiao GC. Intervention analysis with application to economic and environmental problems. J Am Stat Assoc. 1975;70(349):70-9.

45. R Core Team. R: a language and environment for statistical computing, R Foundation for Statistical Computing. 2014. http:// www.R-project.org/. Accessed 27 Feb 2017.

46. DePetris AE, Cook BL. Differences in diffusion of FDA antidepressant risk warnings across racial-ethnic groups. Psychiatr Serv. 2013;64(5):466-71 (471.e1-4).

47. Kales HC, Zivin K, Kim HM, Valenstein M, Chiang C, Ignacio RV, Ganoczy D, Cunningham F, Schneider LS, Blow FC. Trends in antipsychotic use in dementia 1999-2007. Arch Gen Psychiatry. 2011;68(2):190-7.

48. Stevens JR, Jarrahzadeh T, Brendel RW, Stern TA. Strategies for the prescription of psychotropic drugs with black box warnings. Psychosomatics. 2014;55(2):123-33.

49. Dorsey ER, Rabbani A, Gallagher SA, Conti RM, Alexander GC. Impact of FDA black box advisory on antipsychotic medication use. Arch Intern Med. 2010;170(1):96-103.

50. Libby AM, Brent DA, Morrato EH, Orton HD, Allen R, Valuck RJ. Decline in treatment of pediatric depression after FDA advisory on risk of suicidality with SSRIs. Am J Psychiatry. 2007;164(6):884-91.

51. Gibbons RD, Brown CH, Hur K, Marcus SM, Bhaumik DK, Erkens JA, Herings RM, Mann JJ. Early evidence on the effects of regulators' suicidality warnings on SSRI prescriptions and suicide in children and adolescents. Am J Psychiatry. 2007;164(9):1356-63.

52. Bradford WD, Kleit AN. Impact of FDA actions, DTCA, and public information on the market for pain medication. Health Econ. 2015;24(7):859-75.

53. Dusetzina SB, Higashi AS, Dorsey ER, Conti R, Huskamp HA, Zhu S, Garfield CF, Alexander GC. Impact of FDA drug risk communications on health care utilization and health behaviors: a systematic review. Med Care. 2012;50(6):466-78.

54. Kulik A, Bykov K, Choudhry NK, Bateman BT. Non-steroidal anti-inflammatory drug administration after coronary artery bypass surgery: utilization persists despite the boxed warning. Pharmacoepidemiol Drug Saf. 2015;24(6):647-53.

55. Kim HM, Chiang C, Kales HC. After the black box warning: predictors of psychotropic treatment choices for older patients with dementia. Psychiatr Serv. 2011;62(10):1207-14.

56. Friedman RA. Antidepressants' black-box warning - 10 years later. N Engl J Med. 2014;371(18):1666-8.

57. Kruik-Kolloffel WJ, van der Palen J, Kruik HJ, van Herk-Sukel MP, Movig KL. Prescription behavior for gastroprotective drugs in new users as a result of communications regarding clopidogrel -proton pump inhibitor interaction. Pharmacol Res Perspect. 2016;4(4):e00242.

58. Qato DM, Trivedi AN, Mor V, Dore DD. Disparities in discontinuing rosiglitazone following the 2007 FDA safety alert. Med Care. 2016;54(4):406-13.

59. Grimshaw JM, Thomas RE, MacLennan G, Fraser C, Ramsay CR, Vale L, Whitty P, Eccles MP, Matowe L, Shirran L, Wensing M, Dijkstra R, Donaldson C. Effectiveness and efficiency of guideline dissemination and implementation strategies. Health Technol Assess. 2004;8(6):iii-iv (1-72).

60. Canadian Agency for Drugs and Technologies in Health. Rx for change. 2011. https://www.cadth.ca/rx-change. Accessed 24 Feb 2017.

61. Elraiyah T, Gionfriddo MR, Murad MH. Acting on black box warnings requires a GRADE evidence table and an 
implementation guide: the case of teriparatide. J Clin Epidemiol. 2015;68(6):698-702.

62. Sathasivam S, Lecky B. Statin induced myopathy. BMJ. 2008;337:a2286.

63. Aronson JK. Balanced prescribing - principles and challenges. $\mathrm{Br}$ J Clin Pharmacol. 2012;74(4):566-72.

64. Scott IA, Gray LC, Martin JH, Mitchell CA. Minimizing inappropriate medications in older populations: a 10-step conceptual framework. Am J Med. 2012;125(6):529.e4-537.e4.
65. Ikezuagu M, Yang E, Daghstani A, Kaelber DC. Implementing black box warnings (BBWs) in health information systems: an organizing taxonomy identifying opportunities and challenges. Appl Clin Inform. 2012;3(1):124-34.

66. Yu DT, Seger DL, Lasser KE, Karson AS, Fiskio JM, Seger AC, Bates DW. Impact of implementing alerts about medication black-box warnings in electronic health records. Pharmacoepidemiol Drug Saf. 2011;20(2):192-202. 